\title{
Crystallization in settling mixtures of colloidal spheres and plates
}

\author{
S. M. Oversteegen, C. Vonk, J. E. G. J. Wijnhoven, and H. N. W. Lekkerkerker \\ Van 't Hoff Laboratory for Physical and Colloid Chemistry, Debye Research Institute, Utrecht University, \\ P. O. Box 80051, 3508 TB Utrecht, The Netherlands \\ (Received 18 August 2004; revised manuscript received 15 December 2004; published 29 April 2005)
}

\begin{abstract}
The behavior of mixtures of silica spheres and smaller silica-coated gibbsite plates is studied by means of confocal microscopy. Addition of plates slows down the crystallization of the settling spheres. Liquidlike microphases of the plates are found in the sediments of the spheres. It is argued that this is due to simultaneous sedimentation of the plates and spheres as well as depletion interaction between both species. Typical length scales in the sediments, derived from Fourier transforms of the confocal images, suggest there are still specific interactions present.
\end{abstract}

DOI: 10.1103/PhysRevE.71.041406

PACS number(s): 82.70.Dd, 89.75.Fb, 81.16.Dn, 81.16.Be

\section{INTRODUCTION}

Many industrial products, e.g., paints or engineering fluids, contain colloidal particles of different sizes and shapes. The mutual asymmetry of these colloids can induce a net attraction between like-shaped particles by the so-called depletion effect [1-3]. Hence, it may greatly influence the phase stability. Many theoretical and experimental studies have demonstrated this depletion effect for, e.g., bimodal mixtures of colloidal spheres [4], mixtures of spheres and polymers [5,6], platelets and polymers [7-9], and rods and platelets [10-12]. However, experimental studies of mixtures of colloidal spheres and plates are relatively unexplored despite their wide range of applications. Platelike particles are for instance frequently added as rheology enhancers to formulations containing spherical pigments and they may drastically change the way the pigments can be processed.

A platelet cannot assume all conformations when confined between two hard spheres closer to each other than its diameter. The plates gain orientational realization probabilities, which is entropically favorable, when they are depleted from the gap between the two spheres. The resulting density decrease of plates between the spheres gives rise to an unbalanced osmotic pressure which forces the latter together. The strength of this "attraction through repulsion" or depletion potential $W$ reads up to first order in the plate density $\phi_{p}$ $[13,14]$

$$
\begin{aligned}
\frac{W(h)}{k_{B} T}= & -\frac{2}{3} \phi_{p} \frac{\sigma}{L}\left\{\frac{3}{2} \frac{h}{D} \arcsin \frac{h}{D}-\frac{3}{4} \pi \frac{h}{D}\right. \\
& \left.+\left[1+\frac{1}{2}\left(\frac{h}{D}\right)^{2}\right] \sqrt{1-\left(\frac{h}{D}\right)^{2}}\right\} .
\end{aligned}
$$

Here $h$ is the face-to-face distance of the spheres of diameter $\sigma$, whereas $L$ represents the thickness of the plate, $D$ its diameter, and $k_{B} T$ the thermal energy. For low volume fractions this depletion potential turns out to be surprisingly accurate from calculations [14-16].

The net attraction of the spheres may be large enough to induce phase separation above certain concentrations of plates. This has been predicted by means of the free-volume theory [17] applied to spheres and plates [18]. Experiments have not confirmed this predicted phase behavior yet but may nevertheless have great importance to the abovementioned applications. Since we are now able to make stable colloidal mixtures of spheres and plates, we present a closer look at such mixtures here.

\section{EXPERIMENT}

Silica spheres and silica-coated gibbsite plates are synthesized. Van der Waals interactions are likely to be negligible when the spheres are dispersed in dimethylformamide (DMF) since the refractive indices of silica and DMF are closely matched. Mixtures of the silica (coated) particles in DMF are suitable to be examined in real space by means of confocal laser scanning microscopy (CSLM) [19] by incorporating dyes in the particles. Residual charges are screened by the addition of little salt.

In Sec. II A we first describe how the spheres and plates are synthesized and analyzed. Subsequently, we specify in Sec. II B how the samples are prepared.

\section{A. Synthesis and analysis}

\section{FITC labeled silica spheres}

Fluorescein isothiocyanate (FITC, for fluorescence, Fluka) is chemically bound to 3-(aminopropyl)triethoxysilane (APS, purum $>98 \%$, Fluka) where the latter will act as a coupling agent between silica and the dye [20]. Typically $10^{6}$ FITC-APS dimers $[21,22]$ are incorporated into a silica core following the well-known Stöber synthesis [23] using tetraethoxysilane (TEOS, purum $>98 \%$, Fluka). The size of the core of a base catalyzed Stöber synthesis can be chosen by adding the appropriate amount of ammonia (25\% p.a., Merck) [24,25]. We used 9.8 vol \% of $25 \%$ aqueous ammonia in ethanol (p.a., Merck). The synthesized cores were analysed using transmission electron microscopy (TEM). We find that the cores are $656 \pm 42 \mathrm{~nm}$ in diameter.

Subsequently, a thin shell is grown around the prepared cores to reach a desired size. To that end, the appropriate amount of TEOS is added [26,27]. The final particles were analyzed by means of both static light scattering (SLS) and 

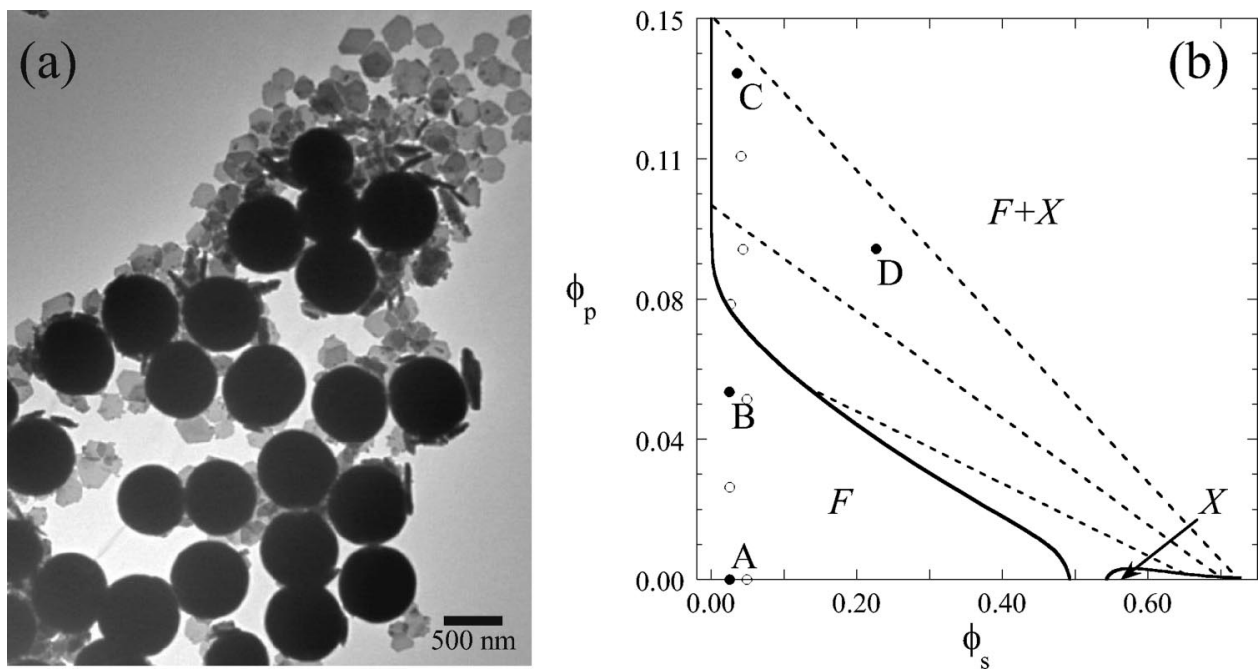

FIG. 1. (a) Transmission electron microscope graph of a mixture of silica-coated (unfluorescent) gibbsite plates and silica spheres. (b) Calculated phase diagram for a mixture of spheres $(\sigma=700 \mathrm{~nm})$ with disks $(D=229 \mathrm{~nm}, L=56 \mathrm{~nm})$ [18]. The dashed tie lines connect volume fractions of spheres $\phi_{s}$ and plates $\phi_{p}$ in the fluid $(F)$ and crystal $(X)$ phase of spheres. The symbols indicate the initial position of the prepared samples, i.e., the overall volume fractions prior to sedimentation. Henceforth, the samples labeled $A$ to $D$, the solid symbols, are considered and represent the characteristics of the neighboring samples.

TEM. From SLS data gathered on a FICA 50 photometer thermostated at $25^{\circ} \mathrm{C}$, we obtained a diameter of $\sigma$ $=723 \pm 36 \mathrm{~nm}$. We finally found by measuring 136 particles on TEM micrographs the spheres to be $\sigma=700 \pm 44 \mathrm{~nm}$ in diameter.

\section{Silica-coated gibbsite plates}

The plates are prepared by coating gibbsite plates with silica. For the gibbsite $\left[\gamma-\mathrm{Al}(\mathrm{OH})_{3}\right]$ synthesis we adopted the method as developed by Wierenga et al. [28]. A mixture of aluminum iso-proxide ( $>98 \%$, Acros) and aluminum secbutoxide $(>95 \%$, Fluka) is dissolved in acidified demineralized water and treated at $85{ }^{\circ} \mathrm{C}$ for three days. Subsequently, the dispersion is dialyzed against demineralized water for one week to eliminate free ions and alcohols formed during the hydrolysis. In order to reduce polydispersity, small particles are removed by centrifugation for $18 \mathrm{~h}$ at $1100 \mathrm{~g}$ [29].

We chose a Stöber-like synthesis to cover the particles with silica. To prevent flocculation of the gibbsite plates in the reaction mixture, containing ethanol and ammonia, polyvinyl pyrrolidone (PVP) is added. This also facilitates the binding of silica onto the surface [30]. After stirring for one day with PVP (40 kDa, Sigma), the dispersion is transferred to ethanol by means of centrifugation. Subsequently, $40 \mathrm{ml} / \mathrm{l}$ of $25 \%$ aqueous ammonia is added with TEOS to grow the silica shell [29]. From the area of 83 hexagon-shaped platelets on TEM images the diameter was found to be $D$ $=229 \pm 25 \mathrm{~nm}$, whereas their thickness of $L=56 \pm 8 \mathrm{~nm}$ was determined from 71 platelets.

Also fluorescent plates were prepared by coating with rhodamine B isothiocynate (RITC, for fluorescence, Fluka). To that end, APS has been coupled to RITC [31] and the dimers have been added to the last step of the silica coverage [32]. After that another portion of TEOS is added to grow a last shell that prevents any specific interactions of the dye and APS. Thus, bare gibbsite platelets of $180 \pm 41 \mathrm{~nm}$ in diameter were coated with an $18 \mathrm{~nm}$ fluorescent silica layer.

\section{B. Sample preparation}

The spheres and nonfluorescent plates are transferred from the solvent mixture of the Stöber synthesis (water, ethanol, and ammonia) into DMF (99\%, Acros) with $1 \mathrm{mM}$ $\mathrm{LiCl}(99 \%$, Acros, reagent ACS crystals) by repeated centrifugation. The fluorescent plates are transferred into DMF by means of vacuum distillation [32]. The densities of the solvent $(\mathrm{DMF}+1 \mathrm{mM} \mathrm{LiCl})$ and particles were determined on an Anton Paar DMA 5000 density meter. The density of the solvent is close to the one in the literature for pure DMF: $948 \mathrm{~kg} / \mathrm{m}^{3}$ and $944 \mathrm{~kg} / \mathrm{m}^{3}$, respectively. We measured (viscosity corrected) densities of the prepared core-shell silica spheres $\left(2099 \pm 5 \mathrm{~kg} / \mathrm{m}^{3}\right)$, the silica-coated plates $\left(2013 \pm 57 \mathrm{~kg} / \mathrm{m}^{3}\right)$, and the fluorescently labeled silicacoated plates $\left(1342 \pm 30 \mathrm{~kg} / \mathrm{m}^{3}\right)$.

The volume fractions of the stock dispersions have been determined by drying and weighing known amounts of the spheres and plates, using the previously measured densities. The stock solution of the core-shell silica spheres in DMF+ $1 \mathrm{mM} \mathrm{LiCl}$ looks clear and orange and fully sediments within a day. The dispersion of the silica-coated plates is turbid and white. The dispersion of the RITC-labeled silicacoated plates looks turbid and pink. The dispersion of spheres is stable against flocculation after two years since it can easily be re-dispersed after sedimentation. The same applies to the dispersion of the silica-coated plates after one year. This suggests that the van der Waals attractions may indeed be neglected.

Approximately $1 \mathrm{ml}$ samples of desired volume fractions were weighed from the stock dispersions and DMF $+1 \mathrm{mM}$ $\mathrm{LiCl}$ into a small vial from which the bottom was replaced by a microscope cover slip of $0.11 \mathrm{~mm}$ thickness using sili- 
con glue (GE Silicons, RTV102 white). After vigorous shaking the vial was placed onto a Nikon Eclipse TE2000-U (inverted) microscope in fluorescence mode, using a Nikon Eclipse C1 scan head and a $100 \times$ oil objective with a numerical aperture of 1.4. A $488 \mathrm{~nm} \mathrm{Ar-Kr}$ laser is used to excitate FITC and a $543 \mathrm{~nm} \mathrm{He}-\mathrm{Ne}$ laser for excitation of RITC. All confocal images are sized $512 \times 512$ pixels and taken in $1.114 \mathrm{~s}$ (top to bottom).

\section{RESULTS}

\section{A. Mixtures of spheres with silica-coated platelets}

A transmission electron microscopy picture is given in Fig. 1(a) for a dried mixture of the silica-coated gibbsite platelets $(D=229 \mathrm{~nm}$ and $L=56 \mathrm{~nm})$ and the FITC-labeled silica spheres $(\sigma=700 \mathrm{~nm})$. From free-volume theory $[17,18]$ an estimated phase diagram for a mixture of hard spheres and disks is given in Fig. 1(b) using the aforementioned experimental values. Coexisting fluid $(F)$ and crystal $(X)$ phases of the spheres are connected by the slanted tie lines.

The overall volume fraction of spheres has to be taken rather low to prevent jamming of the crystallization of the colloidal spheres during sedimentation. We studied ten samples with an initial overall volume fraction of spheres ranging from $\phi_{s}=0.025$ to 0.23 . The initial volume fraction of plates varied from $\phi_{p}=0$ to 0.14 . The initial overall compositions of the samples are indicated by the symbols in Fig. 1. Given the systematic change of the sample behavior, we will discuss only four of those samples here that represent all characteristics best. These samples are indicated $A-D$ in Fig. 1 as filled symbols on their initial position in the phase diagram, i.e., immediately after redispersion. Sample $A$ represents the samples with no plates, whereas sample $B$ shows the behavior of the samples in the predicted one-phase area $(F)$ and the one located on the binodal. The results of the samples in the two-phase region $(F+X)$ close to the binodal are represented by sample $C$ and the sample deep into the two-phase is given by $D$.

The first $75 \mathrm{~min}$ of sedimentation after redispersion are displayed in Fig. 2 for each of the four selected samples, imaged $10 \mu \mathrm{m}$ from the bottom of the vial. Since the particle density has become too dense in this solvent to acquire a clear image at the same height, images of the sediments after one month were taken $5 \mu \mathrm{m}$ from the bottom of the vial and are shown in Fig. 3.

Sample $A$ consists only of spheres $\left(\phi_{s}=0.0251\right)$ that gradually sediment. Within 45 min the system becomes crystalline, as can be seen in the left column of Fig. 2. After one month the sediment has become a single crystal on the scale of $100 \times 100 \mu \mathrm{m}^{2}$, as can be inferred from the upper left image of Fig. 3. The nice sixfold symmetry of the Fourier transform of the image below it confirms this observation.

Adding small amounts of plates $\left(\phi_{p}=0.0502\right)$ to the spheres $\left(\phi_{s}=0.0245\right)$ slows down the sedimentation in sample $B$. Crystalline areas appear after $1 \mathrm{~h}$ as found in the second column of Fig. 2. In a period of one month, the crystalline areas have not reorganized themselves and the patches are preserved, as shown in the second image from the left of
Fig. 3. The powderlike "diffraction" pattern of its Fourier transform with a distinct ring affirms the presence of crystalline patches. Other samples that originate in the presumed one-phase region of the phase diagram (cf. Fig. 1) display similar features.

Samples $\quad C\left(\phi_{p}=0.1354, \phi_{s}=0.0351\right)$ and $D\left(\phi_{p}\right.$ $\left.=0.0885, \phi_{s}=0.2258\right)$ are presumed to reside in the twophase region of the phase diagram. However, no onset of phase separation is observed. The newly formed sediments look disordered or glasslike up to $75 \mathrm{~min}$, as derived from the outer right columns in Fig. 2. In sample $D$ holes are clearly formed within half an hour. These can also be observed in sample $C$ at other spots of the sample after $75 \mathrm{~min}$. In sample $C$ the disordered structures can reorganize themselves somewhat within one month, as can be concluded from the third column from the left in Fig. 3, where still a powderlike Fourier transform is found. Although tiny crystalline patches of spheres can be found after $75 \mathrm{~min}$, the sediment in sample $D$ seems to be fully glasslike after one month. The Fourier transform of the latter does not give any indication of structure.

The Fourier transforms of the sediments after one month, as displayed in Fig. 3, provide us with a typical length scale in the system. Radial means have been determined from five Fourier transformed images for the sediments in samples $A-D$ and are given in Fig. 4. All peaks of samples $A-C$ coincide at $k=1.50 \mu \mathrm{m}^{-1}$. Clearly, this peak has vanished for sample $D$ but due to the form factor still a decay of the intensity at that wave vector is found. This suggests that there is a common length scale present in all samples, corresponding to a typical lattice spacing $d=\frac{1}{2} \sigma \sqrt{3}$. From this we deduce for the (effective) sphere diameter $\sigma_{\mathrm{FFT}}=770 \mathrm{~nm}$.

\section{B. Mixture of spheres and dyed platelets}

The recently obtained RITC-labeled plates [32] turn out yet to be unstable at higher volume fractions. We obtained a stock solution of only $\phi_{p}=0.0061$, which makes a detailed study as in the previous section inaccessible. However, the behavior of plates in the sediments can be pinned down. Since the size of the plates are in the same order as the optical resolution of the microscope, they cannot be observed individually. Clusters of plates, however, can be detected.

We put a mixture of dyed plates and spheres to rest for a couple of hours. The FITC and RITC emissions were imaged simultaneously in two separate channels. The left image of Fig. 5 gives the FITC channel in green and is similar to the results as previously found in the sediment of sample $C$. Although part of the FITC emission (spheres) is also detected by the RITC channel in the middle of Fig. 5, clear clusters appear that were not visible in the FITC channel. The combination of both channels gives the third image of Fig. 5, where the red clusters clearly fill the holes observed in the FITC channel.

The sediment is studied at several heights in the sample. Images on the bottom of the vial and 10 and $20 \mu \mathrm{m}$ above it are shown from left to right, respectively, in Fig. 6. On the bottom the clusters imaged red seem quite rigid and are consistent with aggregates of plates only [32]. The number of 


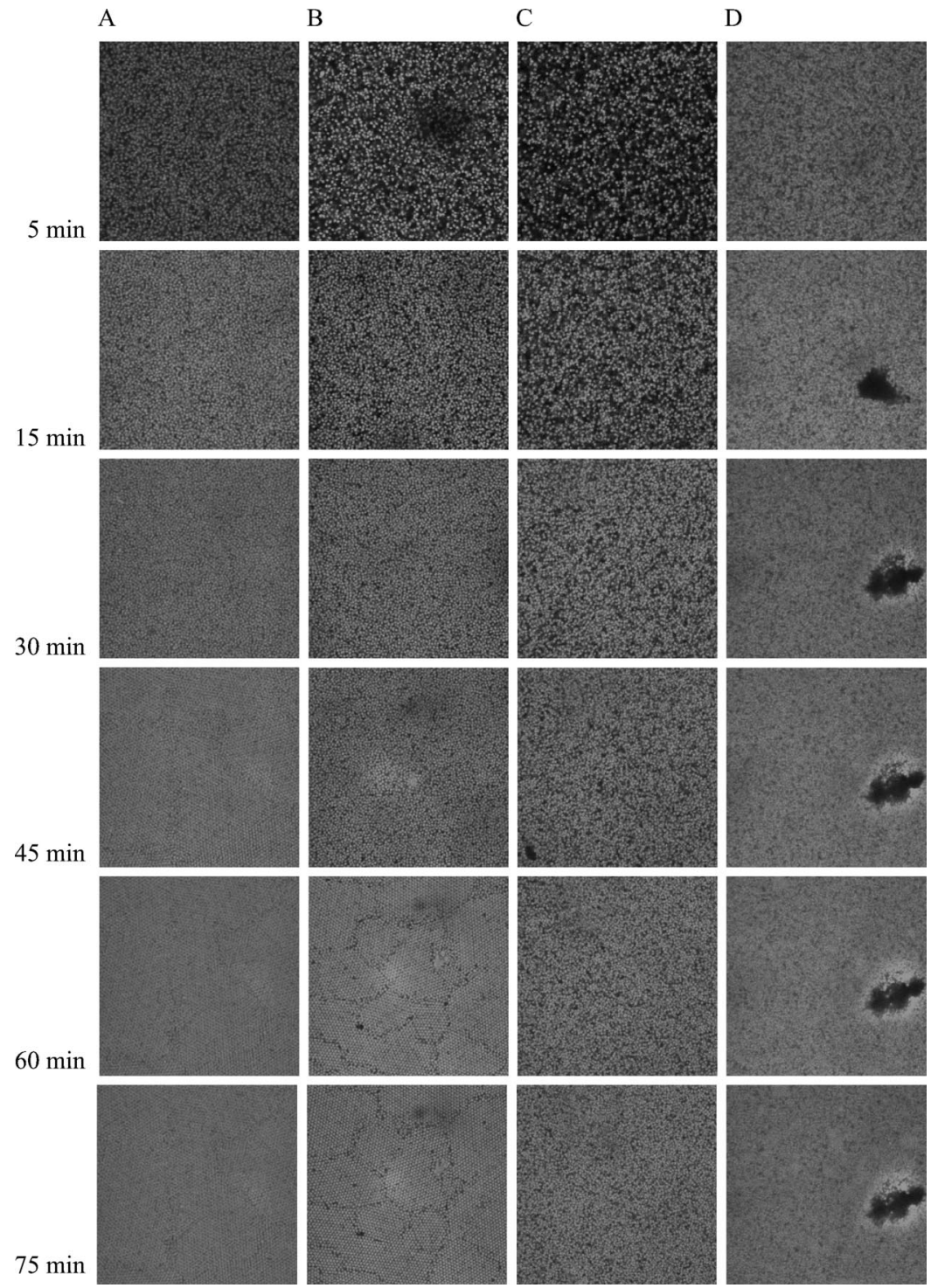

FIG. 2. Confocal images of the samples $A-D$ (left to right, cf. Fig. 1) $10 \mu \mathrm{m}$ from the bottom of the vial 5, 15, 30, 45, 60, and 75 min (top to bottom) after redispersion. The images are $50 \times 50 \mu \mathrm{m}^{2}$ each.

red structures is increasing with increasing height. Moreover, higher in the sample the structures are relatively dynamic compared to the movement of the spheres.

\section{DISCUSSION}

At a certain height of the sample, the density increases due to sedimentation. In sample A the spheres thus slowly enter the two-phase region of the phase diagram, where it forms crystalline sediments. By adding plates, the biphasic gap between the fluid $(F)$ and crystal $(X)$ in Fig. 1 opens up. Hence, we expected that with increasing plate concentration, crystallization is found earlier since the phase-separating region is entered sooner upon sedimentation. This effect has indeed been observed for sphere-polymer $[5,33]$ and sphererod mixtures [25]. However, the settling rate seems to slow down significantly with increasing plate concentration as may be conjectured from the decreasing number of spheres in the first row in Fig. 2. Since the buoyant mass of the particles does not change, this is likely because of a rise in the viscosity at increasing volume fraction of particles. This effect has also been observed in dispersions of spheres [34,35], sphere-polymer mixtures [5], and sphere-rod mixtures $[25,36,37]$. The experiments on the colloidal mixtures support our idea that the viscosity of the samples increases faster than the increase in depletion attraction [cf. Eq. (1.1)] can overcome. Yet, given the little material we have, we are not able to conduct reliable measurements of the viscosity of the samples.

The sediments of samples $B, C$, and $D$ seem consistent with the notion that the spheres are quenched into the phaseseparation region with increasing plate concentration. Crystalline or glasslike regions of spheres are formed and are not able to reorganize into a single crystal after a month. Moreover, the depleted plates are trapped in the sediments of 
A
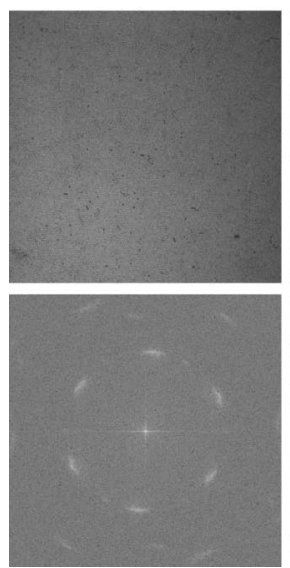

B
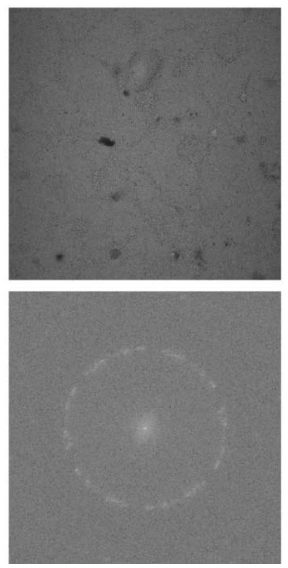

$\mathrm{C}$
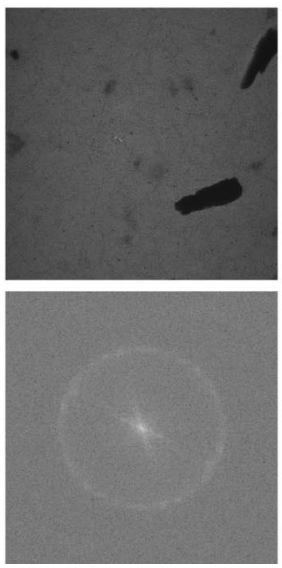

$\mathrm{D}$
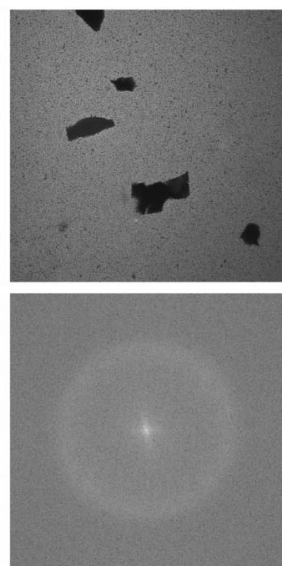

FIG. 3. Confocal images of the sediments one month after redispersion $5 \mu \mathrm{m}$ from the bottom of the vial in real space (top) and Fourier space (bottom). From left to right the samples $A-D$ as indicated in Fig. 1. The top images are $100 \times 100 \mu \mathrm{m}^{2}$ each.

samples $C$ and $D$ and form microphases rather than a macroscopic phase separation. These microphases appear as holes in the sediments of samples $B-D$, as these are not fluorescent. The existence of microphases is confirmed by adding fluorescently labeled plates, where RITC emission is found in these holes. They appear as dynamical regions in Fig. 5, suggesting a liquidlike microphase of the plates.

In addition to their depletion, sedimentation of the plates is also substantial. A measure for the decay of the concentration as a function of height is the so-called gravitational length

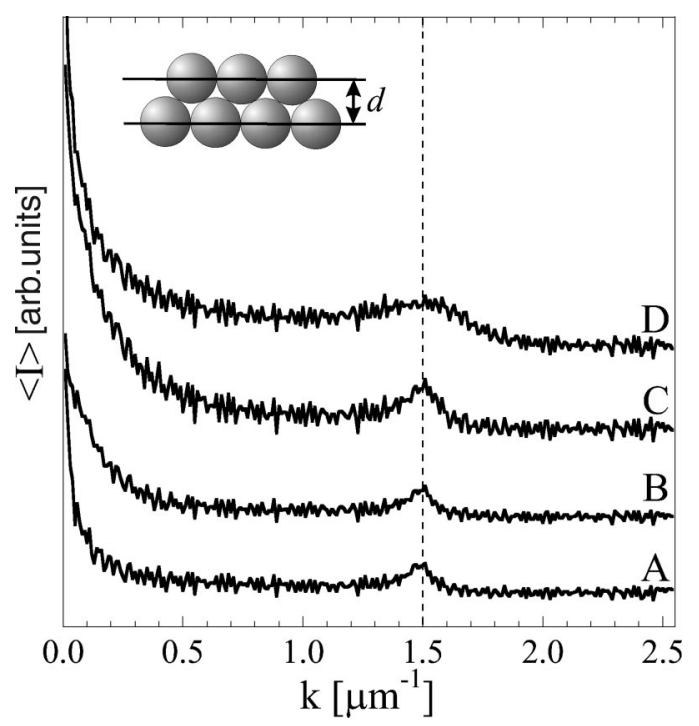

FIG. 4. Radial averages of the intensity $\langle I\rangle$ of four Fourier transforms of the sediments of which one is depicted in Fig. 3 for the samples $A-D$ in Fig. 1 (bottom to top). From the peak, indicated by the dashed line, the effective interparticle distance is determined to be $770 \mathrm{~nm}$. For the aggregating sample $D$ the structure factor has vanished but the form factor is still clearly present.

$$
\ell \equiv \frac{k_{B} T}{\Delta \rho v_{p} g}
$$

where $\Delta \rho$ is the buoyancy-corrected density of the particle, $v_{p}$ the particle's volume, and $g$ the standard acceleration of free fall. Applying the above experimental values, we find for the spheres a gravitational length of $\ell_{s}=2.0 \times 10^{-6} \mathrm{~m}$ and for the plates $\ell_{p}=0.88 \times 10^{-4} \mathrm{~m}$. Although the sphere sediment front is smaller, that of the plates is quite small as compared to the gravitational lengths of polymers or rods that are several times the height of the sample. This may be another explanation why no explicit phase separation is observed: considerable sedimentation of the plates takes place while depleting. This can be gathered from Fig. 6, where many of the platelets are found within $20 \mu \mathrm{m}$ from the bottom of the sample. Unfortunately, we could not image much deeper into the sample since the platelets scatter a lot. This problem also prevents us from going higher up in the phase diagram. By the naked eye it is seen that the vial containing dyed plates consists of two parts: an orange sediment and a pink, turbid supernatant that becomes clearer in time owing to sedimentation of the plates.

The scattering of the silica-coated gibbsite plates may be reduced by acid-leaching them [29]. The thus obtained hollow silica plates are better refractive index matched. The leached hollow plates will in addition also have a lower buoyant mass, which may reduce the problem of simultaneous sedimentation of both types of particles while phase separating as mentioned previously. However, leaching is a very delicate procedure where the platelets pass their isoelectric point twice, which makes them very vulnerable to aggregation. Moreover, when colored plates are used, the dye may be affected by the acid.

The effective mass of the spheres can be lowered by coating the particles with a polymer and using a density matched solvent. However, this may introduce other interactions and different optical properties. Progress can be made by using 

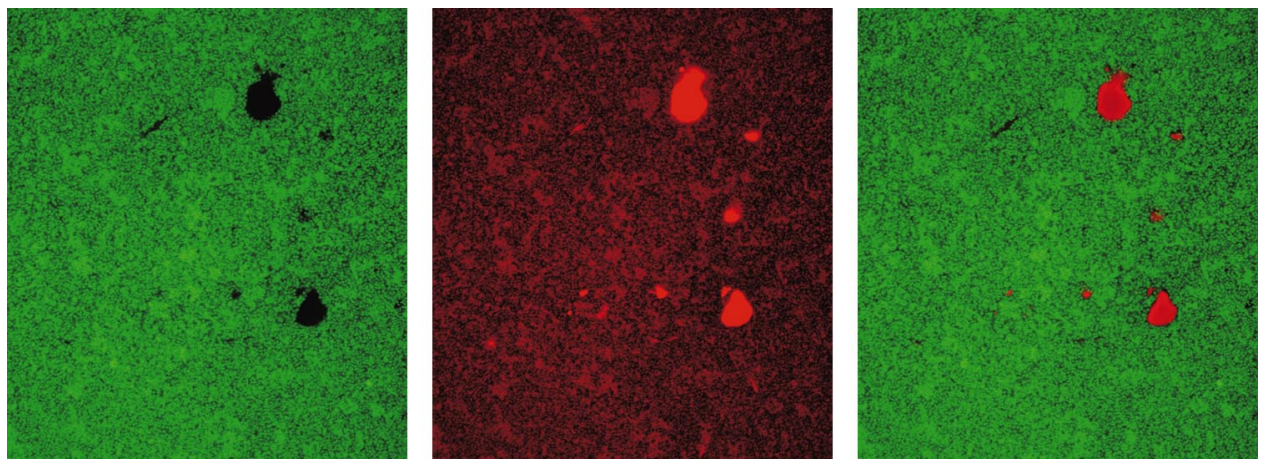

FIG. 5. (Color) Confocal image $\left(100 \times 100 \mu \mathrm{m}^{2}\right)$ of a mixture of FITC-labeled spheres and RITC-labeled plates. The left picture images the FITC emission channel and is represented in green. The RITC emission channel, given in red in the middle, also detects some of the FITC emission. The dual-detection image is shown on the right hand side.

poly(methylmethacrylate) (PMMA) spheres in a mixture of organic solvents [38]. Such a model system for hard spheres is almost matched with respect to both density and refractive index. However, no PMMA-coated plates are available yet. No examples are known of direct deposition of PMMA on gibbsite. Although PMMA on a silica surface is possible [39], deposition on silica-coated gibbsite plates will decrease the aspect ratio considerably.

Another way to diminish the influence of settling is by using small sample height, e.g., by horizontally oriented capillaries. This has previously been done for mixtures of spheres with rods $[36,37]$ but the increasing viscosity prevented to go to higher rod concentrations that could be achieved in the vials [25], like the ones used in this study. Hence, the aforementioned difficulties that may arise due to the presumed increase in viscosity may be reinforced by using capillaries in order to decrease sedimentation.

A typical intersphere spacing has been deduced from the intensities of the Fourier transformed confocal images, Fig. 4. If we assume (random) close packing, the volume fraction in the sediments ought to be

$$
\phi_{s}=\frac{\pi \sqrt{2}}{6}\left(\frac{\sigma_{\mathrm{SLS}}}{\sigma_{\mathrm{FFT}}}\right)^{3}=0.6130 .
$$

This volume fraction is in agreement with the one predicted from free-volume theory in the crystal (cf. Fig. 1). The di- ameter of the spheres derived from the Fourier transforms, $\sigma_{\mathrm{FFT}}=770 \mathrm{~nm}$, differs from the bare diameter in solution, $\sigma_{\mathrm{SLS}}=723 \mathrm{~nm}$. This difference may be contributed partially to the presence of residual charges on the spheres. However, since it has been found that the thickness of the double layer is only about $10 \mathrm{~nm}$ [21], maybe other repulsive interactions are present as well. This makes the calculated phase diagram, based on hard interactions only, merely a crude first order estimate for the free-energy landscape that may act as a template for the dynamic system [40].

\section{CONCLUSIONS}

From free-volume theory we calculated a phase diagram that may act as a rough estimate for the free-energy landscape the settling mixture of spheres and plates traverses. In contrast to sphere-polymer and sphere-rod mixtures, volume fractions of the plates in the vicinity of the binodal slow down early crystalline ordering of the spheres. This, however, still leads to more grain boundaries and more powderlike structures. In the presumed two-phase region the disordered sediment structures are kinetically arrested and this obstructs (further) crystallization. Moreover, in the biphasic region fluidlike microphases of plates are trapped in the sediment. We argue that these microphases are partially due to
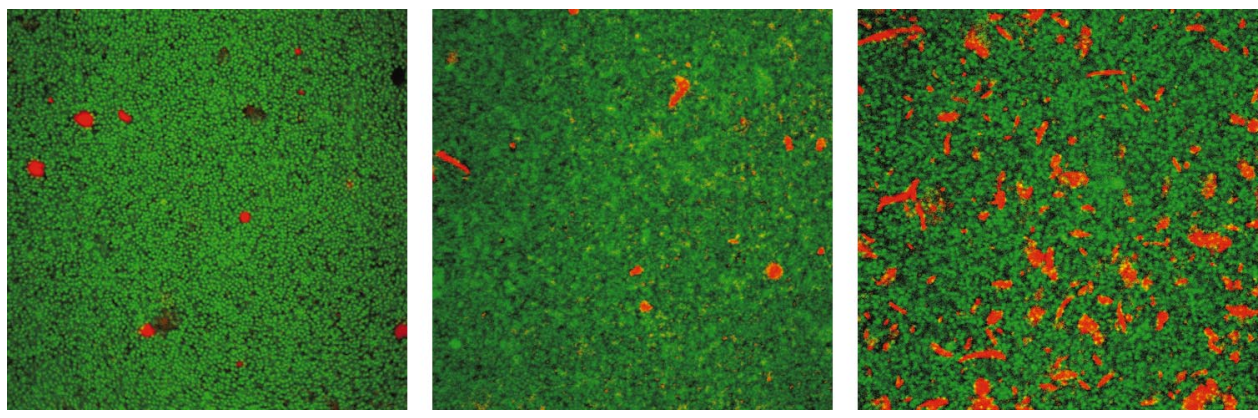

FIG. 6. (Color) Confocal micrographs $\left(100 \times 100 \mu \mathrm{m}^{2}\right)$ of a mixture of dyed spheres and plates, imaged 0 , 10, and $20 \mu \mathrm{m}(\mathrm{left}$ to right) from the bottom of the vial, respectively. The FITC emission (spheres) is represented as green, whereas the RITC emission (plates) is given in red. 
depletion interactions and partially because of simultaneous sedimentation such that the plates are subsequently confined between the dense spheres. These effects prevent us from finding a possible path the system passes through the phase diagram during sedimentation. Decreasing the buoyant mass of the species may open up such studies in the future.

\section{ACKNOWLEDGMENTS}

The work of SMO is part of the SoftLink research programme of the "Stichting voor Fundamenteel Onderzoek der Materie (FOM)," which is financially supported by the "Nederlandse Organisatíe voor Wetenschappelijk Onderzoek (NWO)."
[1] S. Asakura and F. Oosawa, J. Chem. Phys. 22, 1255 (1954).

[2] S. Asakura and F. Oosawa, J. Polym. Sci. 32, 183 (1958).

[3] A. Vrij, Pure Appl. Chem. 48, 471 (1976).

[4] M. Dijkstra, R. van Roij, and R. Evans, Phys. Rev. E 59, 5744 (1999), and references therein.

[5] E. H. A. de Hoog, W. K. Kegel, A. van Blaaderen, and H. N. W. Lekkerkerker, Phys. Rev. E 64, 021407 (2001).

[6] R. Tuinier, J. Rieger, and C. G. de Kruif, Adv. Colloid Interface Sci. 103, 1 (2003), and references therein.

[7] M. A. Bates and D. Frenkel, Phys. Rev. E 62, 5225 (2000).

[8] F. M. van der Kooij, M. Vogel, and H. N. W. Lekkerkerker, Phys. Rev. E 62, 5397 (2000).

[9] S. D. Zhang, P. A. Reynolds, and J. S. van Duijneveldt, Mol. Phys. 100, 3041 (2002).

[10] F. M. van der Kooij and H. N. W. Lekkerkerker, Langmuir 16, 10144 (2000).

[11] H. H. Wensink, G. J. Vroege, and H. N. W. Lekkerkerker, J. Chem. Phys. 115, 7319 (2001).

[12] S. Varga, A. Galindo, and G. Jackson, Phys. Rev. E 66, 011707 (2002).

[13] M. Piech and J. Walz, J. Colloid Interface Sci. 232, 86 (2000).

[14] S. M. Oversteegen and H. N. W. Lekkerkerker, Phys. Rev. E 68, 021404 (2003).

[15] L. Harnau and S. Dietrich, Phys. Rev. E 69, 051501 (2004).

[16] S. M. Oversteegen and H. N. W. Lekkerkerker, Physica A 341, 23 (2004).

[17] H. N. W. Lekkerkerker, W. C. K. Poon, P. N. Pusey, A. Stroobants, and P. B. Warren, Europhys. Lett. 20, 559 (1992).

[18] S. M. Oversteegen and H. N. W. Lekkerkerker, J. Chem. Phys. 120, 2470 (2004).

[19] A. van Blaaderen, A. Imhof, W. Hage, and A. Vrij, Langmuir 8, 1514 (1992).

[20] A. van Blaaderen and A. Vrij, Langmuir 8, 2921 (1992).

[21] A. Imhof, A. van Blaaderen, and J. K. G. Dhont, Langmuir 10, 3477 (1994).

[22] A. Imhof and J. K. G. Dhont, Phys. Rev. E 52, 6344 (1995).
[23] W. Stöber, A. Fink, and E. Bohn, J. Colloid Interface Sci. 26, 62 (1968).

[24] G. H. Bogush, M. A. Tracy, and C. F. Zukoski IV, J. NonCryst. Solids 104, 95 (1988).

[25] S. M. Oversteegen, J. E. G. J. Wijnhoven, C. Vonk, and H. N. W. Lekkerkerker, J. Phys. Chem. B 108, 18158 (2004).

[26] H. Giesche, J. Eur. Ceram. Soc. 14, 205 (1994).

[27] H. Giesche, in Surfactant Science Series, edited by T. Sugimoto, (Marcel Dekker, New York, 2000), Vol. 92, pp. 126146.

[28] A. M. Wierenga, T. A. J. Lenstra, and A. P. Philipse, Colloids Surf., A 134, 359 (1998).

[29] J. E. G. J. Wijnhoven, Chem. Mater. 16, 3821 (2004).

[30] C. Graf, D. Vossen, A. Imhof, and A. van Blaaderen, Langmuir 19, 6693 (2003).

[31] N. A. M. Verhaegh and A. van Blaaderen, Langmuir 10, 1427 (1994).

[32] C. Vonk, S. M. Oversteegen, and J. E. G. J. Wijnhoven, J. Colloid Interface Sci. (to be published).

[33] V. J. Anderson and H. N. W. Lekkerkerker, Nature (London) 416, 811 (2002).

[34] G. K. Batchelor, J. Fluid Mech. 52, 245 (1972).

[35] W. B. Russel, D. A. Saville, and W. R. Schowalter, Colloidal Dispersions (Cambridge University Press, Cambridge, U.K., 1989), Chap. 12.

[36] G. H. Koenderink, G. A. Vliegenthart, S. G. J. M. Kluijtmans, A. van Blaaderen, A. P. Philipse, and H. N. W. Lekkerkerker, Langmuir 15, 4693 (1999).

[37] G. A. Vliegenthart, A. van Blaaderen, and H. N. W. Lekkerkerker, Faraday Discuss. 112, 173 (1999).

[38] P. N. Pusey and W. van Megen, Nature (London) 320, 340 (1986).

[39] W. K. Kegel and A. van Blaaderen, Science 287, 290 (2000).

[40] R. M. L. Evans, W. C. K. Poon, and F. Renth, Phys. Rev. E 64, 031403 (2001). 